\title{
Access to human, animal, and environmental journals is still limited for the One Health community ${ }^{\star}$ EC
}

\author{
Carol E. Vreeland, DVM, MLS, AHIP; Kristine M. Alpi, MLS, MPH, AHIP; Caitlin A. Pike, MLS, AHIP; \\ Elisabeth E. Whitman, MS; Suzanne Kennedy-Stoskopf, DVM, PhD, Dipl. ACZM
}

See end of article for authors' affiliations.

DOI: http://dx.doi.org/10.3163/1536-5050.104.2.003

\begin{abstract}
Objective: "One Health" is an interdisciplinary approach to evaluating and managing the health and well-being of humans, animals, and the environments they share that relies on knowledge from the domains of human health, animal health, and the environmental sciences. The authors' objective was to evaluate the extent of open access (OA) to journal articles in a sample of literature from these domains. We hypothesized that OA to articles in human health or environmental journals was greater than access to animal health literature.
\end{abstract}

Methods: A One Health seminar series provided fifteen topics. One librarian translated each topic into a search strategy and searched four databases for articles from 2011 to 2012. Two independent investigators assigned each article to human health, the environment, animal health, all, other, or combined categories. Article and journal-level OA were determined. Each journal was also assigned a subject category and its indexing evaluated.

Results: Searches retrieved 2,651 unique articles from 1,138 journals; 1,919 (72\%) articles came from 406 journals that contributed more than 1 article. Seventy-seven $(7 \%)$ journals dealt with all 3 One Health domains; the remaining journals represented human health $487(43 \%)$, environment 172 $(15 \%)$, animal health $141(12 \%)$, and other/combined categories $261(23 \%)$. The proportion of OA journals in animal health (40\%) differed significantly from journals categorized as human (28\%), environment (28\%), and more than 1 category (29\%). The proportion of OA for articles by subject categories ranged from $25 \%-34 \%$; only the difference between human (34\%) and environment (25\%) was significant.

Conclusions: OA to human health literature is more comparable to animal health than hypothesized. Environmental journals had less OA than anticipated.

Keywords (Medical Subject Headings): Publishing, Periodicals as Topic, Access to Information, Veterinary Medicine, Environment, Environmental Health, Medicine

"One Health" is an integrated, transdisciplinary approach to solve complex problems at the diverse interfaces shared by humans, animals, and the environment [1]. The One Health approach to evaluating and managing the health and well-being of humans, animals, and the environments that they share relies on knowledge from the domains of

* Based on a poster presented at MLA'13, the 113th Annual Meeting of the Medical Library Association; Boston, MA; May 7, 2013.

Supplemental Appendix A, Appendix B, Appendix C, Table 2, and Table 4 are available with the online version of this journal. human health, animal health, and the environmental sciences. Although there is a growing body of literature about the development of the One Health concept as documented by Pepper, Carrigan, Shurtz, and Foster [2], this literature is not the same as the combination of literature from the three domains that is applied in service of One Health. Every discipline related to One Health has its unique mindset and language, with corresponding lists of acronyms that are frequently an impediment to effective communication across the participating professions. Relevant papers guiding a One Health 
approach may never specifically use "One Health" as a term or concept.

To promote better communication and collaboration among health professionals and environmental scientists, a public monthly One Health Intellectual Exchange Group (IEG) hosted by the North Carolina Biotechnology Center was launched in 2009. In 2011, faculty from the North Carolina State University College of Veterinary Medicine, University of North Carolina's Gillings School for Global Public Health, Duke Global Health Institute, and Nicholas School of the Environment at Duke University expanded the IEG series into a weekly seminar course with eight One Health focus areas [3]. The eight focus area modules were the following: an introduction to One Health; environmental health and ecology; the human and animal bond; zoonoses and emerging infectious diseases; food and water safety; disease surveillance, informatics, and disaster preparedness; benefits of comparative medicine; and policy and education (Appendix A, online only). Each seminar speaker recommended papers to read prior to the session to provide a foundation for the topic because student backgrounds and majors were quite diverse.

Represented student majors included master's of public health, master's of animal science, doctor of veterinary medicine, graduate-level environmental sciences, and undergraduate-level biochemistry, engineering, and biology.

Open access (OA) to relevant literature is very important to scholars and practitioners working on interdisciplinary problems. The One Health Proof of Concept Workgroup found that few studies assess outcomes in human, animal, and environmental spheres simultaneously [4], making it important to be able to access articles from each of the three domains to get a more complete picture.

The objective of this study was to evaluate the extent of OA to journal articles in a sample of literature relevant to One Health from the human, animal, and environmental domains. Working in a college of veterinary medicine and supporting faculty, staff, and students addressing interdisciplinary problems under the One Health umbrella [5], the authors were familiar with the extent of OA in human biomedical and public health literature and the literature of veterinary medicine but were less familiar with environmental journals. In light of general availability of environmental information and OA to publications such as Environmental Health Perspectives, we thought it likely that environmental literature would be relatively open compared to the other subject areas. Therefore, we hypothesized OA to articles from human health or environmental journals was greater than access to animal health literature. We chose to look at articlelevel subject categorization and access, as well as journal-level categorization and access, because they might differ. Article-level access relates more to authors' decisions about OA for a content domain, while journal-level access and subject categorization are driven by publishers and associations.

Understanding the distinction and having data would inform our efforts to promote increased OA to this literature.

\section{METHODS}

\section{Identifying the sample of unique articles}

Fifteen of the seminar topics for the spring 2012 course were selected as a source of examples in One Health to be converted into representative literature for this study (Appendix A, online only). Those not selected included One Health overviews and core competencies, presentations on specific projects like the US Agency for International Development's PREDICT, and generic topics for which we already had searched specific examples. For example, we did not search "Pollutants and Environmental Health," because we had already searched "Mercury Dynamics in Aquatic Systems." A health sciences librarian with a veterinary degree (Vreeland) translated each of the fifteen selected seminar topics into a search strategy, using seminar reviews on the course blog to assist with keyword selection (Appendix B, online only), and ensured that all the papers cited by the seminar presenter were retrieved by the searches. After consulting with the course cocoordinator (Kennedy-Stoskopf) to confirm the search strategy, the librarian searched using the strategy across a group of databases relevant to One Health over July 2012 to October 2012. The databases chosen for the search were BIOSIS Previews, CAB Abstracts, PubMed, and Web of Science, because they are some of the most used databases in medicine, animal health, and environmental sciences available through our institutional subscriptions. All search results were limited to journal articles from 2011 to 2012. Although some topics lend themselves to specialty databases such as Canary for animals as sentinels or PsycINFO for animal abuse and family violence, we only present data from databases that covered all One Health topics. 


\begin{tabular}{|c|c|c|c|c|}
\hline Category & \# of journals & $\%$ of total & \# of articles & $\%$ of total \\
\hline Human Health $(\mathrm{H})$ & 487 & $42.8 \%$ & 1,021 & $38.5 \%$ \\
\hline Environment $(\mathrm{E})$ & 172 & $15.1 \%$ & 530 & $20.0 \%$ \\
\hline Animal Health $(A)$ & 141 & $12.4 \%$ & 491 & $18.5 \%$ \\
\hline All $(\mathrm{H}, \mathrm{E}$, and $\mathrm{A})$ & 77 & $6.8 \%$ & 308 & $11.6 \%$ \\
\hline Both $\mathrm{H}$ and $\mathrm{A}$ & 51 & $4.5 \%$ & 95 & $3.6 \%$ \\
\hline Both $\mathrm{H}$ and $\mathrm{E}$ & 38 & $3.3 \%$ & 8 & $0.3 \%$ \\
\hline Both $E$ and $A$ & 15 & $1.3 \%$ & 7 & $0.3 \%$ \\
\hline Other & 157 & $13.8 \%$ & 191 & $7.2 \%$ \\
\hline Total & 1,138 & $100 \%$ & 2,651 & $100 \%$ \\
\hline
\end{tabular}

Table 1

Subject categories for journals and articles

All 2011-2012 journal article citations resulting from each of the 15 topics were downloaded into RefWorks and labelled with the source database information. The citations were exported from RefWorks to Excel and then manually examined for duplicates across topics and source databases.

Duplicates were collapsed into unique article records $(n=2,651)$ listing all of the source databases in which that article was found in our search results. Due to the variability in search processing across the databases searched, we also checked to see whether each journal contributing articles to this pool was reported to be indexed in the source databases, based on the indexing coverage of the journal listed in the Ulrichsweb.com (Ulrich's) global serials directory as of 2013. The combination of indexing from the search results combined with the journal-level indexing reported by Ulrich's was used to represent database indexing coverage of the journals in this study.

\section{Unique article subject classification}

Two independent investigators assigned each unique article to a broad subject category representing the areas of One Health: "human," "animal," or "environment." Articles that represented all three areas were coded as "all," and those that represented a combination of two of the three subjects were coded as a combination of those two subjects (Table 1 ). These combined categories were analyzed separately but collapsed into "more than one category" for data display. There was also a category of "other." Definitions of the subject categories used by the coders are provided in online only Appendix C.

Different pairs of independent investigators reviewed the various topics. Each independent reviewer was blind to the other's classifications until the initial concordance rates were calculated. Article categorization concordance rates ranged from 58\% for the topic "Neem" to $96 \%$ for the topic "Dalfampridine," with an average rate of agreement of $80 \%$ across the 15 topics. All discordant items were reviewed by a third investigator and a decision made about how to categorize the article.

\section{Journal subject categorization}

The unique articles came from 1,138 unique journals. Two independent investigators assigned each journal to a broad subject category, using the same categories and process described above. The only difference was that the categorization was based on the journal's description of its subject coverage on its website or in Ulrich's and did not take into consideration any of the unique article categorizations. The journal concordance rates for initial categorization was $86 \%$.

The subject assignment process for journals in tropical diseases, food, and microbiology was especially complex. Tropical and emerging infectious diseases journals were coded as all on the presumption that human cases often arise from vectors in the environment or on animals. Food journals about protection, hygiene, and control were coded as all; journals mostly about food chemistry, science, and so on were coded as other (Food). Clinical microbiology journals were coded as human, unless veterinary specified, in which case they were coded as animal. Applied microbiology journals were coded all unless otherwise specified; any other microbiology was coded as other (Micro). Further specifics on journal-level coding are in online only Appendix C. Subject assignment for the thirty 


\begin{tabular}{lrcc|}
\hline \multicolumn{1}{c}{ Country } & \# of journals & \% open access & \% partial access \\
\hline United States & 307 & $14.7 \%$ & $14.3 \%$ \\
United Kingdom & 259 & $14.7 \%$ & $10.0 \%$ \\
India & 84 & $53.6 \%$ & $1.2 \%$ \\
Netherlands & 71 & $1.4 \%$ & - \\
Germany & 58 & - & $3.4 \%$ \\
China & 39 & $17.9 \%$ & - \\
Brazil & 36 & $91.7 \%$ & - \\
France & 18 & $38.9 \%$ & $11.1 \%$ \\
Italy & 18 & $55.6 \%$ & - \\
Pakistan & 18 & $94.4 \%$ & - \\
Japan & 16 & $50.0 \%$ & $12.5 \%$ \\
Australia & 14 & $42.9 \%$ & $7.1 \%$ \\
Switzerland & 14 & $64.3 \%$ & $7.1 \%$ \\
Poland & 13 & $61.5 \%$ & - \\
South Korea & 12 & $83.3 \%$ & - \\
Kenya & 11 & $100.0 \%$ & - \\
Russia & 10 & - & - \\
Turkey & 10 & $90.0 \%$ & - \\
Canada & 9 & $44.4 \%$ & $33.3 \%$ \\
Nigeria & 8 & $75.0 \%$ & - \\
& & & \\
\hline
\end{tabular}

Table 3

Open access in 2013 for the 20 countries with the most One Healthrelated journals in this data set

journals with the most articles in the dataset appears in Table 2, online only.

\section{Journal indexing, country of publication, and open access coverage}

For each journal, initial information about indexing coverage came from the list of articles in our literature search results. Indexing coverage was then checked in Ulrich's, along with country of publication and full-text OA. For titles not in Ulrich's, information was gathered from the journal website. Ulrich's applies the "Open Access" limiter to publications that are in the Directory of Open Access Journals or otherwise "support a funding model that does not charge readers or their institutions for access" [6]. Because Ulrich's OA description applied only to completely OA, we visited the websites of the publishers of the journals that were not $\mathrm{OA}$ in Ulrich's and looked at the back issues for any information about embargoed full text and captured the embargo length in months. Embargoed access via PubMed Central was considered partial access. In cases where we were not clear about whether the access was open, we tested articles from the journal on a computer that was not connected to the university network.

The final decision for each journal was open for OA, partial for embargoed access, and not for no systematic OA. Access status was ascertained in spring of 2013. For the thirty journals with the most articles, we rechecked the access in February 2015 to see if anything had changed since our prior reporting of the data.

\section{Unique article open access classification}

Individual articles could be OA for a variety of reasons: US government authorship, funder mandate, paid OA fees, or part of special issues funded for or chosen to be OA. We used the PubMed article categorization as "Free Full Text" to import and tag sets of articles in RefWorks. For those not initially labelled "Free Full Text," we followed the PubMed link on a computer outside of the university network to see if free full text of the article was available. If free full text was available from the publisher or from PubMed Central, we coded the specific article as open. Articles that were not freely linked from PubMed were then compared with the journal-level analysis of access as open, partial, or closed. Access to individual articles in non-OA journals was checked by individually searching the title of each article on a personal computer not connected to the university network. Articles were coded as open if they were published (1) in an OA journal, (2) during the open timeframe of an embargoed partial access journal, or (3) as individually open due to author arrangements with the publisher. Articles were coded as partial if they were in the embargoed timeframe of a partial access journal, meaning they would eventually become openly available. Articles were classified as closed if they were published in a closed access journal and no individual-level OA arrangement was evident.

\section{Statistical comparisons}

All statistical tests were performed using Epitools epidemiological calculator [7]. The proportions of journals indexed by each of the four databases were compared using a chi-square test for two rows and four columns, followed by $z$ tests to compare two sample proportions. The proportions of OA journals classified as human, animal, environment, all, other, and the variations on more than one category were compared using a chi-square test for eight rows and three columns, using Epitools epidemiological calculator. Significant findings were further compared using $z$ tests to compare two sample proportions. Comparisons of sample proportions 


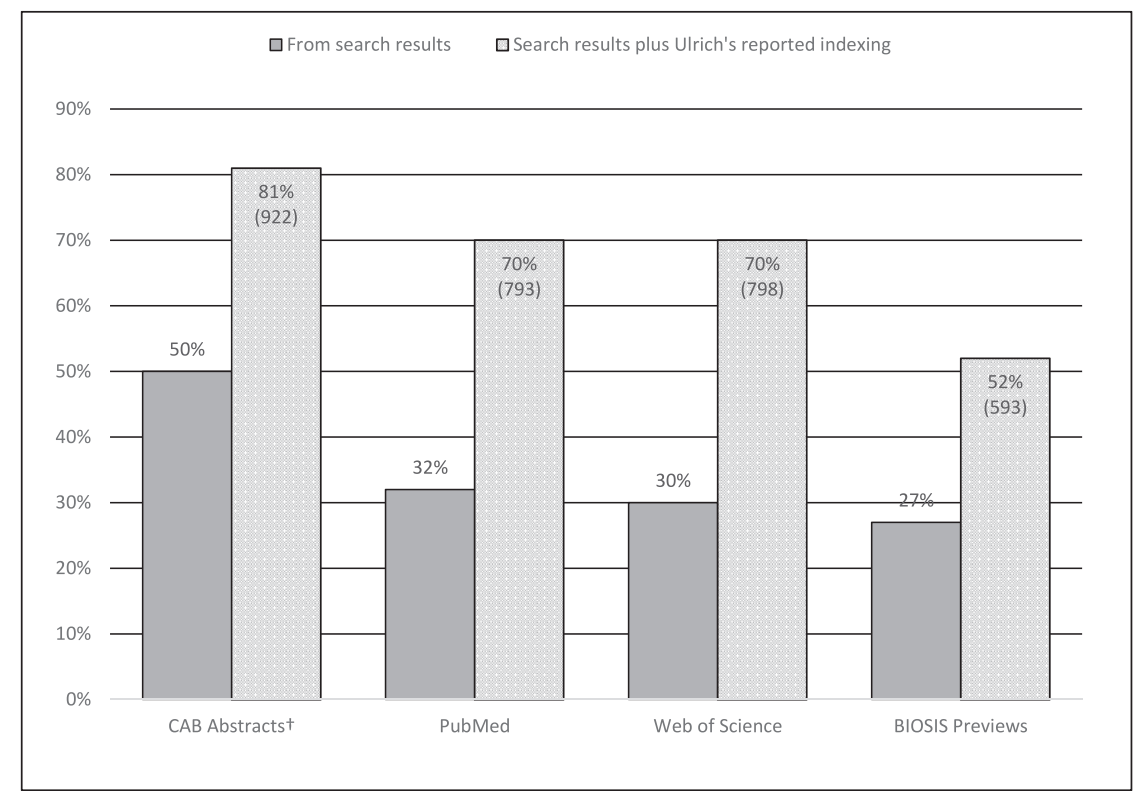

\section{Figure 1}

Database indexing coverage ${ }^{\star}$ of the One Health journals in this data set $(n=1,138)$

${ }^{*}$ As of 2013.

† The difference between CAB Abstracts and both PubMed and Web of Science was statistically significant $(p<0.0001)$, as were the differences between those three databases and BIOSIS Previews.

were performed for OA availability of articles by subject category.

\section{RESULTS}

Searches retrieved 2,651 unique articles from 1,138 journals. As shown in Table 1, there were more human health journals and articles than any other subject category, followed by environment and then animal health. Only 77 (6.8\%) journals and 308 (11.6\%) articles dealt with all 3 domains that One Health comprises. The 406 journals with more than 1 unique article represented 1,919 (72.4\%) of the retrieved articles, and an additional 732 journals had a single citation. Database indexing coverage of journals in this study, based on the combination of search results and Ulrich's reported indexing, ranged from $52 \%$ in BIOSIS Previews to $81 \%$ in $\mathrm{CAB}$ Abstracts (Figure 1), which was much higher than the rates found when relying solely on the search results to indicate coverage. The difference between the proportions of indexing coverage between $\mathrm{CAB}$ Abstracts and both PubMed and Web of Science was statistically significant, $z=6.1, p<0.0001$, as were the differences between those 3 databases and BIOSIS Previews.
Journal-level and article-level open access by subject category

There was a statistically significant difference between the proportions of OA journals by subject category (Figure 2) (chi-square statistic 42.04, $d f=14$, $p=0.0001$ ). Further comparisons using $z$ tests for 2 sample proportions between each of the subject categories showed that the proportion of journals found to be OA in the animal health category $(40 \%)$ differed significantly from the OA proportion of journals categorized as human $(28 \%, p=0.0065)$, environment $(28 \%, p=0.0251)$, and more than one category $(29 \%, p=0.0385)$. These OA percentages did not include the partial access (in 2013), also shown in Figure 2 for these journals across all subject categories. The embargo time ranged from 2-60 months, with a mean of 14.4 months and standard deviation of 9.2 months.

Article-level OA by subject category (Figure 3) showed a statistically significant difference in proportions between subjects (chi-square statistic 75.56, $d f=14, p<0.0001$ ). However, when testing between groups using $z$ tests comparing sample proportions, the only significant difference found in proportion of OA to articles categorized by each subject was between human $(34 \%)$ and environment $(25 \%$, $p=0.0003$ ). The proportion of OA in articles was fairly 


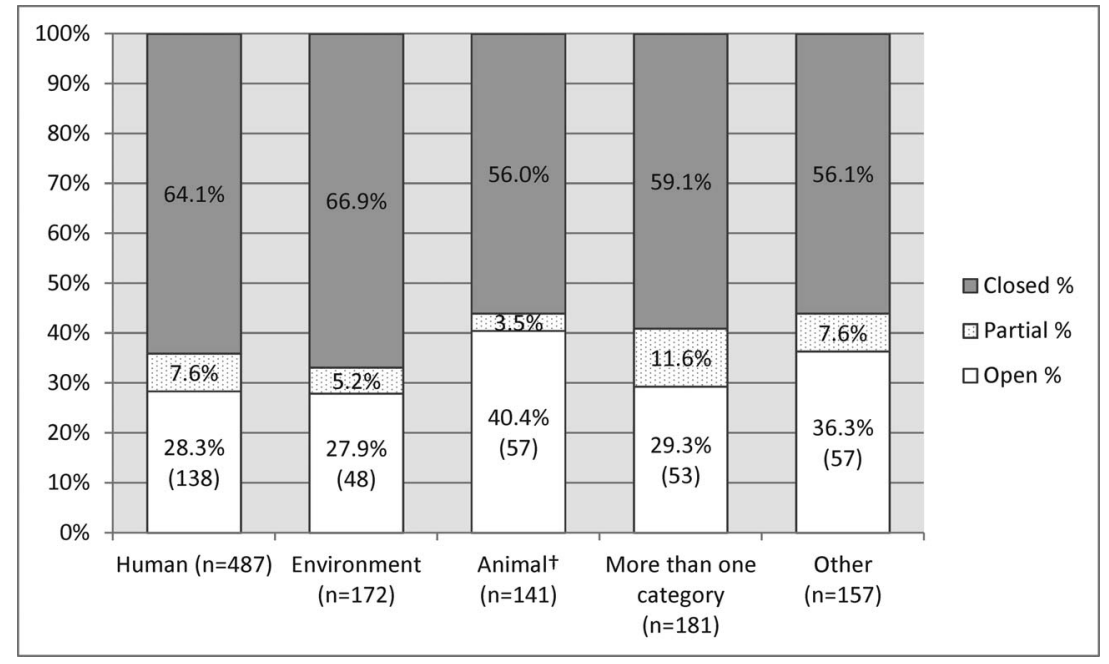

\section{Figure 2}

Journal-level open access $(O A)^{*}$ by subject category $(n=1,138)$

* As of 2013.

† Animal proportion of $O A$ differs significantly from Human $(p=0.0065)$, Environment $(p=0.0251)$, and More than one category proportions $(p=0.0385)$.

consistent: the remaining categories ranged from $30 \%$ for animal to $32 \%$ for other, results that were not statistically different from those for human and environment.

Comparing the percentages of $\mathrm{OA}$ in each subject category at both the journal level and the article level showed mostly non-significant differences going in both directions, with the exception of the human and animal health percentages. In animal health, the decrease from $40.4 \%$ in journals to $29.5 \%$ in articles was statistically significant $(z=2.4, p=0.0144)$.

Conversely in human health, there was a statistically significant increase from $28.3 \%$ in journals to $34.0 \%$ in articles $(z=2.2, p=0.0267)$.

Top twenty countries publishing journals with One Health content

The 1,138 unique journals were published in 62 different countries. The percentage of OA in the 20 countries with the most One Health journals in this data set (Table 3) varied from being $100 \%$ open for the 11 journals published in Kenya to completely closed for the 10 journals published in Russia.

\section{Access status of top thirty journals with the most unique articles}

Table 2, online only, shows the access status of the top 30 journals with the most unique articles in this data set. From the initial study in 2013 until rechecked February 19, 2015, only 2 of these top 30 journals changed access status $(n=31$ due to a tie at position 30). The Journal of Pharmacy Research is no longer published by Elsevier as of 2014. Now published by JPR Solutions (Mohali, Punjab, India), it appears to be an OA journal, though the publisher had not confirmed as of April 13, 2015, that this is the long-term intention for the journal. The Journal of Veterinary Internal Medicine, published by Wiley for the American College of Veterinary Internal Medicine, became OA in 2015 [8].

\section{DISCUSSION}

The breadth of literature relevant to One Health is demonstrated by the 1,138 journals in this study retrieved from searches on only 15 One Health topics. Our objective was to examine the extent of OA to journal articles in this sample of literature drawn from the human, animal, and environmental domains. We found human and animal health literature varied by whether OA was measured in terms of access to articles, where human health was a higher proportion (Figure 3), or access to journals, where there was more OA in animal health, and the differences between subject categories were statistically significant (Figure 2). As the articles were fairly closely tied to the 15 topic searches, the journal-level access provides a broader view of access in the 


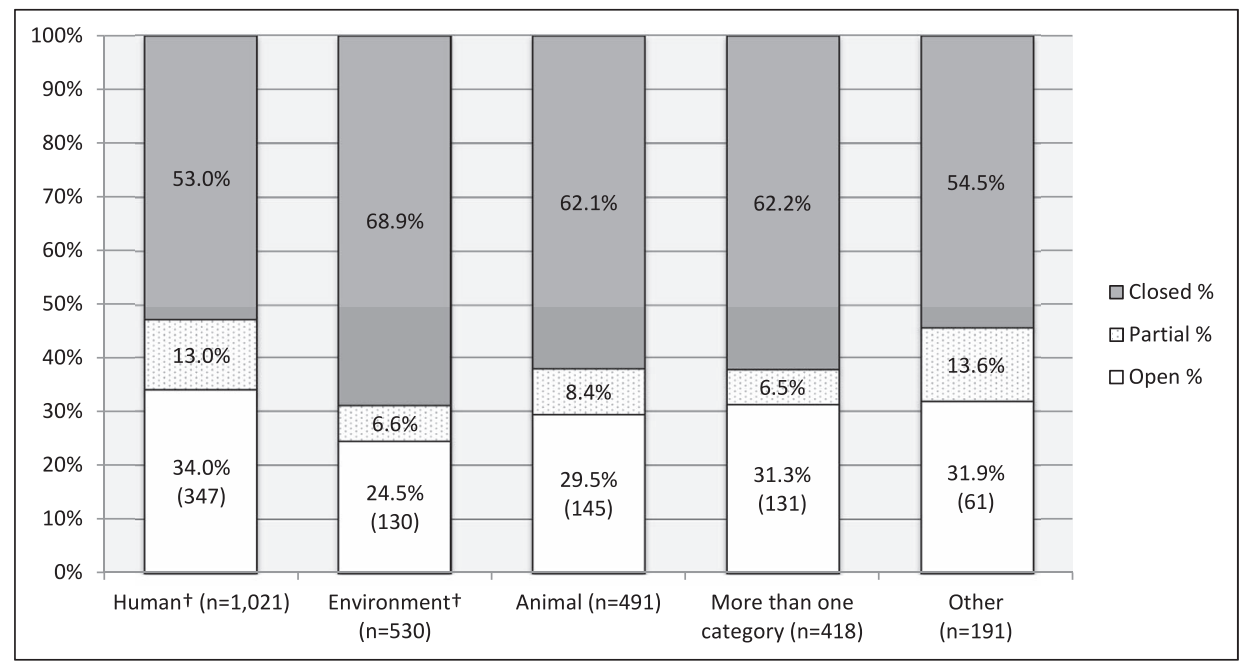

Figure 3

Article-level $O A^{*}$ by subject category $(n=2,651)$

${ }^{*}$ As of 2013.

† The only statistically significant difference in OA between the proportion of article-level OA in these categories is between Human and Environment $(p=0.0003)$.

domains. Regardless of the method used, fewer than half of journals or articles across all domains in this study were openly accessible. Our hypothesis of greater access to human health and environmental literature than to animal health depends on whether access is compared proportionally or in absolute numbers. Defining greater as a larger proportion of OA within the journals assigned to the subject category, animal health has a higher proportion of OA than human or environmental literature. However, considering absolute number of $\mathrm{OA}$ journals by subject category (Figure 2), there are 138 human OA titles, 57 animal OA titles, and 48 environment OA titles. Depending on the point of view, human or animal health journals could be leading in OA. Where our hypothesis was clearly incorrect is on the state of OA to environmental articles and journals related to these select One Health topics: environmental journals did not have as much access as anticipated.

Article-level OA for animal health (29.5\%) for our sample from 2011-2012 was very similar to the $27 \%$ OA for veterinary articles identified by Nault by searching veterinary school publications in 20062007 [9]. To provide a comparison to support our journal-level findings, in August 2015, we used the 2013 Journal Citation Reports (JCR) data from Thomson Reuters to examine the rates of OA in journals in several categories relevant to One Health. OA in JCR is defined as the journal being present in the Directory of Open Access Journals. There were no exact equivalents to any of our subject categories, but several related categories are worth reporting. Rates of OA in JCR were much lower for both

"Environmental Sciences," 13 OA out of 216 journals $(6.0 \%)$, and "Environmental Studies," 3 OA out of 98 $(3.1 \%)$, than the rate of $28 \%$ we found. For

"Veterinary Sciences" (26 OA/132 journals), 20\% is lower than our $40 \%$ finding for animal health, but part of animal health is also addressed in "Agriculture, Dairy \& Animal Science," which had a rate of $23.1 \%$ (12 OA/52 journals). Human health varied between $19.3 \%$ for "Public, Environmental \& Occupational Health" (59 OA/305 journals); 30.1\% for "Medicine, General \& Internal" (47 OA/156 journals); and 45.5\% for "Tropical Medicine" (10 OA/ 22 journals), creating a sense that our overall finding of $28 \%$ for human health was reasonable. Minimal differences of $1 \%-4 \%$ were seen when repeating the queries with the 2014 JCR data, suggesting little change at the journal level in OA from 2013 to 2014.

\section{Limitations}

The topics that we searched in some cases found articles from journals specific to those topics. This 
also likely influenced the list of countries with the most One Health-related journals in our findings. Neurology, for example, had twelve articles, which is likely a function of the search on the approach to comparative medicine for spinal cord injuries in dogs and humans. If a different comparative medicine topic had been presented, another leading specialty journal might have made the list instead. For countries, an example is the topic "Neem Tree: Nature's Bio-Defense at Its Best"; those search results were highly represented in journals published in India, which ranked third in the list of countries with the most journals in the data set. The search strategies used were created by a single librarian; other strategies might produce different results. The results reported here are from literature at least four years before this study is published. For example, the Journal of Veterinary Internal Medicine is now completely OA; however, there were no changes in OA from 2013 to 2015 for twenty-nine of the thirtyone journals with the most unique articles. Subject assignments and certain other classifications necessary in the methods represent the judgment of the authors.

Ulrich's reports a much higher percentage of database indexing of the 1,138 journals in our data set than was indicated by our literature search results (Figure 1). There are several possible reasons for this. A database's indexing of a particular journal might only be partial, so that potentially not all articles relevant to our searches would be included in our search results. In some cases (e.g., some smaller international journals), it takes a long time for new journal issues to get indexed by a particular database. We ran our literature searches in 2012 and limited our results to articles published in 2011 or 2012. Perhaps if we had chosen or included older articles, our search-related journal indexing percentage rates would have been closer to what Ulrich's lists. It is also possible that error was introduced in getting data from Ulrich's. Anticipating questions about indexing in free databases other than PubMed, we did a post-study analysis comparing the 315 journals not indexed by PubMed to the list of journals indexed in Agricola and found that Agricola covered $45 \%(n=142)$ of those journals in 2013. We focused the database coverage component on databases available at the NCSU Libraries, which was why we did not assess Scopus coverage. We can provide our unique journal or article lists, should anyone wish to assess Scopus coverage.

\section{Next steps}

We plan to share these published results with the editors of many of the journals included in this data set, in the hopes of raising awareness about the importance of open and embargoed partial access to One Health article topics among the diverse scholars and practitioners needing access to One Health literature. Open access to human, animal, and environmental journals is key to promoting One Health collaborations. A recent focus article in Science Translational Medicine calls for a "One Literature" approach to facilitate communication among scientists, physicians, and veterinarians; raise cross-species awareness; and bring together new research communities and collaborations [10]. In our opinion, advocacy to editorial boards and authors of high-article-count journals covering all three domains, such as EcoHealth (seventy-nine citations), that do not provide OA is crucial to improving access to One Health knowledge. The list of journal titles (Table 4, online only) may be useful to libraries wishing to consider strengthening or assessing their collections to support education, research, and practice for those taking a One Health approach to interdisciplinary problems.

\section{ACKNOWLEDGMENTS}

We appreciate the assistance of the public service assistants at the William R. Kenan, Jr. Library of Veterinary Medicine, who assisted with data entry and link checking.

\section{AUTHORS' CONTRIBUTIONS}

Carol E. Vreeland, Kristine M. Alpi, and Caitlin A. Pike designed the study. Suzanne Kennedy-Stoskopf provided course materials. Vreeland, Alpi, Pike, and Elisabeth E. Whitman conducted the study and were involved in the analysis and interpretation of data. Alpi performed statistical analyses. All authors contributed to the manuscript and have given final approval of the paper.

\section{REFERENCES}

1. Centers for Disease Control and Prevention. One Health [Internet]. The Centers; 2013 [cited 20 Apr 2015]. $<$ http://www.cdc.gov/onehealth/>.

2. Pepper C, Carrigan E, Shurtz S, Foster MJ. Exploring librarian roles in support of One Health. J Agric Food Inf. 
2013;14(4):321-33. DOI: http://dx.doi.org/10.1080/ 10496505.2013.826582.

3. Barrett MA, Bouley TA, Stoertz AH, Stoertz RW. Integrating a One Health approach in education to address global health and sustainability challenges. Front Ecol Environ. 2011;9(4):239-45.

4. Kachani M, Rabinowitz P. Proof of concept workgroup. In: Stone Mountain Workgroups [Internet]. Centers for Disease Control and Prevention; Apr 2012 [cited 20 Apr 2015]. <http://www.cdc.gov/onehealth/pdf/workgroups/ newsletter-april-2012.pdf $>$.

5. Lackey M, Alpi K. Librarians' contributions to a One Health course across three universities. Paper presented at: MLA '13, 113th Annual Meeting of the Medical Library Association; Boston, MA; May 3-8, 2013. 6. Q: Ulrich's includes open access journals. what does that mean? [Internet] Serials Solutions; 2013 [cited 15 Jan 2013]. <http://www.ulrichsweb.com/ulrichsweb/faqs. asp\#About_Ulrichs $>$.

7. Sergeant ESG. Epitools epidemiological calculators [Internet]. AusVet Animal Health Services and Australian Biosecurity Cooperative Research Centre for Emerging Infectious Disease; 2013 [cited 1 Mar 2013]. <http:// epitools.ausvet.com.au $>$.

8. Research Information. Wiley converts three more journals to open access [Internet]. Europa Science; 2015 [cited 4 Feb 2015]. <http://www.researchinformation. info/news/news_story.php?news_id=1831>.

9. Nault AJ. Open access of publications by veterinary faculty in the United States and Canada. J Vet Med Educ. 2011;38(1):33-41.

10. Christopher MM. One health, one literature: weaving together veterinary and medical research. Sci Transl Med. 2015 Sep;7(303):303fs36. DOI: http://dx.doi.org/10.1126/ scitranslmed.aab0215.

\section{AUTHORS' AFFILIATIONS}

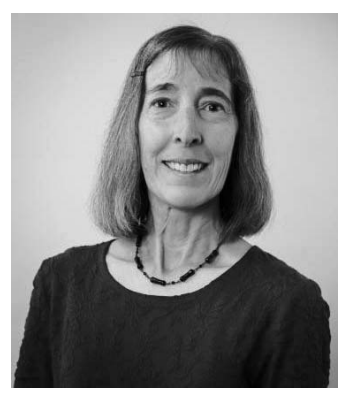

Carol E. Vreeland, DVM, MLS, AHIP, carol_vreeland@ ncsu.edu, Associate Director, William Rand Kenan, Jr. Library of Veterinary Medicine, NCSU Libraries, and Adjunct Assistant Professor, Department of Population Health \& Pathobiology, College of Veterinary Medicine; Kristine M. Alpi, MLS, MPH, AHIP, kristine_alpi@ncsu.edu, Director, William Rand Kenan, Jr. Library of Veterinary Medicine, NCSU Libraries, and Adjunct Assistant Professor, Department of Population Health \& Pathobiology, College of Veterinary Medicine; Caitlin A. Pike, MLS, AHIP, + caitlin.a.pike@gmail.com, Intern, William Rand Kenan, Jr. Library of Veterinary Medicine, NCSU Libraries, College of Veterinary Medicine; Elisabeth E. Whitman, MS, betsy_whitman@ncsu.edu, University Library Technician, William Rand Kenan, Jr. Library of Veterinary Medicine, NCSU Libraries; Suzanne Kennedy-Stoskopf, DVM, PhD, Dipl. ACZM, suzanne_stoskopf@ncsu.edu, Research Professor, Ecosystem Health, Department of Clinical Sciences, College of Veterinary Medicine; North Carolina State University, 1060 William Moore Drive, Raleigh, NC 27607

Received April 2015; accepted November 2015
+ Currently, Caitlin A. Pike, caiapike@iupui.edu, Nursing and Medical Humanities Liaison Librarian, University Library, Indiana University-Purdue University Indianapolis, 755 West Michigan Street, Indianapolis, IN 46202. 